\title{
LISTADO DE EVALUADORES/AS 2014-15
}

Agudo, Yolanda Arroyo - UNED (España)

Agulló Díaz, Camen - Universitat de València (España)

Alarcón Meneses, Luis Alfonso - Universidad del Atlántico (Colombia)

Álvarez Fernández, María - Universidad de Oviedo (España)

Álvarez González, Yasmina - Universidad de La Laguna (España)

Ballarín Domingo, Pilar - Universidad de Granada (España)

Beas Miranda, Miguel - Universidad de Granada (España)

Braster, Sjaak - Erasmus Universiteit Rotterdam (Holanda)

Brizzi, Gian Paolo - Università di Bologna (Italia)

Cagnolati, Antonella - Universita di Foggia (Italia)

Carbone, Graciela - Universidad Nacional de Luján (Argentina)

Carli, Sandra - Universidad de Buenos Aires (Argentina)

Carrillo Gallego, Dolores - Universidad de Murcia (España)

Castillo Gómez, Antonio - Universidad de Alcalá (España)

Colmenar Orzaes, Carmen - Universidad Complutense de Madrid (España)

Costa Rico, Antón - Universidade de Santiago de Compostela (España)

Cuesta Fernández, Raimundo - Fedicaria. Salamanca (España)

Cunningham, Peter - University of Cambridge (Reino Unido)

Diez Velasco, Francisco - Universidad de La Laguna (España)

Escolano Benito, Agustín - Universidad de Valladolid (España)

Espigado Tocino, Gloria - Universidad de Cádiz (España)

Fernández Soria, José Manuel - Universitat de València (España)

Ferraz Lorenzo, Manuel - Universidad de La Laguna (España)

Flecha García, Consuelo - Universidad de Sevilla (España)

Gabriel Fernández, Narciso de - Universidade de A Coruña (España)

Gómez García, María Nieves - Universidad de Sevilla (España)

González Pérez, Teresa - Universidad de La Laguna (España)

Groves, Tamar - Universidad de Extremadura (España)

Guereña, Jean-Louis - Université François Rabelais, Tours (Francia) 
Guichot Reina, Virginia - Universidad de Sevilla (España)

Hernández Díaz, José María - Universidad de Salamanca (España)

Herrera, Martha Cecilia - Universidad Pedagógica Nacional (Colombia)

Jiménez Eguizábal, Alfredo - Universidad de Burgos (España)

López Bausela, José Ramón - Universidad de Cantabria (España)

López Hernández, José - Universidad de Murcia (España)

Luengo Navas, Julián Jesús - Universidad de Granada (España)

Magalhães, Justino - Universidade de Lisboa (Portugal)

Mahamud Angulo, Kira - UNED (España)

Martinez Valle, Carlos - Universidad Complutense de Madrid (España)

Mayordomo Pérez, Alejandro - Universitat de València (España)

McCulloch, Gary - UCL Institute of Education (Reino Unido)

Meda, Juri - Università di Macerata (Italia)

Milito, Cecilia - UNED (España)

Moreno Martínez, Pedro Luis - Universidad de Murcia (España)

Morente Valero, Francisco - Universitat Autònoma de Barcelona (España)

Munakata, Kazumi - Pontifícia Universidade Católica de São Paulo (Brasil)

Naya Garmendia, Luis María - Universidad del País Vasco (España)

Ossenbach Sauter, Gabriela - UNED (España)

Otero Urtaza, Eugenio - Universidade de Santiago de Compostela (España)

Polenghi, Simonetta - Università Cattolica del Sacro Cuore (Italia)

Porto Ucha, Angel Serafín - Universidade de Santiago de Compostela (España)

Pozo Andrés, María del Mar del - Universidad de Alcalá (España)

Ramos Zamora, Sara - UNED (España)

Rabazas Romero, Teresa - Universidad Complutense de Madrid (España)

Rubio Mayoral, Juan Luis - Universidad de Sevilla (España)

Sanchidrián Blanco, Carmen - Universidad de Málaga (España)

Sani, Roberto - Università di Macerata (Italia)

Sanz Díaz, Federico - Universidad de Burgos (España)

Sevilla Merino, Diego - Universidad de Granada (España)

Terrón Bañuelos, Aida - Universidad de Oviedo (España)

Tiana Ferrer, Alejandro - UNED (España)

Valls Montés, Rafael - Universitat de València (España)

Villalaín Benito, José Luis - UNED (España) 